\title{
On the Conduction Mechanism of Silicate Glass Doped by Oxide Compounds of Ruthenium (Thick Film Resistors). 3. The Minimum of Temperature Dependence of Resistivity
}

\author{
Gulmurza Abdurakhmanov \\ The Institute of Power Engineering and Automation, The Uzbek Academy of Sciences, Tashkent, \\ Uzbekistan \\ Email: gulmirzo@mail.ru
}

Received 2 June 2014; revised 5 July 2014; accepted 21 July 2014

Copyright @ 2014 by author and Scientific Research Publishing Inc.

This work is licensed under the Creative Commons Attribution International License (CC BY).

http://creativecommons.org/licenses/by/4.0/

(c) (i) Open Access

\section{Abstract}

This article is the final part of the investigation of conduction mechanism of silicate glass doped by oxide compounds of ruthenium (thick film resistors). In the first part [1], the formation of percolation levels due to diffusion of dopant atoms into the glass has been considered. The diffusion mechanism allowed us to explain shifting of the percolation threshold towards to lower value and the effect of firing conditions as well as the components composition on the electrical conduction of the doped glass. The coexistence of thermal activation and localization of free charge carriers as the result of nanocrystalline structure of the glass was the subject of the second part [2]. Because of it, the resistivity of the doped silicate glass is proportional to exp $\left(-a T^{-\zeta}\right)$ at low temperatures $(T<$ $50 \mathrm{~K}), 0.4<\zeta<0.8$. Structural transitions of nanocrystals take place at high temperatures $(T>800$ $\mathrm{K}$ ) and the conductivity of the doped silicate glass decreases sharply. We consider the origin of the minimum in the temperature dependence of resistivity of the doped silicate glass here. It is shown that the minimum arises from merge of impurity band into the valence band of glass at temperature high enough, so thermal activation of charge carriers as well as its hopping are failed, and scattering of free charge carriers become predominant factor in the temperature dependence of the resistivity.

\section{Keywords}

Lead-Silicate Glass, Thick Film Resistors, Minimum of Resistivity, Doping, Energy Bands, Conductivity, Thermal Activation, Hopping 


\section{Introduction}

This article is the final part of the investigation of conduction mechanism of silicate glass doped by oxide compounds of ruthenium (thick film resistors).

In the first part [1], the formation of percolation levels due to diffusion of dopant atoms into the glass has been considered. The diffusion mechanism allowed us to explain shifting of the percolation threshold towards to lower value and the effect of firing conditions as well as the components composition on the electrical conduction of the doped glass (DSG). The coexistence of thermal activation and localization of free charge carriers as the result of nanocrystalline structure of the glass was the subject of the second part [2]. Because of it, the resistivity of the doped silicate glass is proportional to exp $\left(-a T^{-\zeta}\right)$ at low temperatures $(T<50 \mathrm{~K}), 0.4<\zeta<0.8$. Structural transitions of nanocrystals take place at high temperatures $(T>800 \mathrm{~K})$ and the conductivity of the doped silicate glass decreases sharply.

An "enigmatic" minimum near the room temperature [3]-[10] and metallic conductance beyond it [11] take place in the silicate glass doped by oxide compounds of ruthenium. Adding of other metal oxides does not change qualitatively the situation [9] [12]. The origin of the Minimum is considered here. It is shown that the minimum arises from the merge of impurity subband into the valence band of glass at temperature high enough, so thermal activation of charge carriers as well as its hopping are failed, and scattering of free charge carriers become predominant factor in the temperature dependence of the resistivity.

\section{Experiment}

The manufacturing process of DSG used here is standard for technology of thick film resistors and was described elsewhere (the mixture of the glass and the dopant powders on the alumina substrate have been fired at $T_{\mathrm{f}}=1073-1125 \mathrm{~K}$ in $\left.\tau=10 \mathrm{~min}\right)$. Content of $\mathrm{RuO}_{2}$ in our samples of DSG is indicated in the figure captions. The glass compositions investigated are as follows (weight \%):

Glass1 $\mathrm{SiO}_{2} 27$; $\mathrm{PbO} 67 ; \mathrm{BaO} 4 ; \mathrm{MgO} 2$;

Glass2 $\mathrm{SiO}_{2}$ 32; $\mathrm{PbO} 63 ; \mathrm{Al}_{2} \mathrm{O}_{3}$;

Glass3 $\mathrm{SiO}_{2}$ 33; $\mathrm{PbO} 67$.

Firing temperature $T_{\mathrm{f}}$ is $1073 \mathrm{~K}$ for glass $1,1123 \mathrm{~K}$ for glass 2 and glass 3.

The value of resistance was measured by digital multimeter Sch-300 having error less than $0.2 \%$. The temperature was measured by the same multimeter and standard Pt-PtRh thermocouple. The thermocouple was calibrated in standard temperature points of water boiling (373 K), tin and silver solidification (505 and $1234 \mathrm{~K}$ accordingly).

The DSG samples are screen-printed and have dimensions $10 \times 10 \times 0.025 \mathrm{~mm}^{3}$ so measured values of the resistance $R(\mathrm{Ohm})$ and the resistivity $\rho(\mathrm{Ohm} \cdot \mathrm{cm})$ are related as $\rho=R t=2.5 \times 10^{-3} R$, here $t=2.5 \times 10^{-3} \mathrm{~cm}$ is the thickness of the DSG.

\section{Conduction Mechanisms of DSG}

The effective medium theory [3] [13] can evaluate the measurable (effective) values of permittivity $\varepsilon^{*}$ and conductivity $\rho^{*}$ of the DSG on the basis of known values of same parameters for the dopant and the glass $\left(\varepsilon_{\mathrm{d}}, \rho_{\mathrm{d}}\right.$ and $\varepsilon_{\mathrm{g}}, \rho_{\mathrm{g}}$ accordingly). It is assumed in this theory that the volume content $C$ of the dopant is known a priory. But experiments show that this assumption is not justified for the DSG due to physical or chemical interactions of components at the firing temperatures. These processes can generate new compounds or regions of unknown parameters. On the other hand, the minimum of $\rho(T)$ can arise only from coexisting of at least the two components having opposite temperature dependence of conductivity — dielectric and metallic with same values of conductivity. Unfortunately these values are strong differing in the DSG: $\rho_{\mathrm{g}}>10^{15} \mathrm{Ohm} \cdot \mathrm{cm}$ and $\rho_{\mathrm{d}}<2.5 \times 10^{-4}$ Ohm $\cdot \mathrm{cm}$ (for $\mathrm{RuO}_{2}$, [14] [15]). This fact has the total effect for all the known mechanisms of the DSG conductivity and will be considered more detailed later.

The percolation theory leads to the percolation threshold on the conductivity

$$
\sigma(C)=\sigma_{0}\left(C-C_{\mathrm{c}}\right)^{t}
$$

here $C_{\mathrm{c}}$ is the critical volume content of the dopant (about 16 vol\% in the three dimensions), $t$ is the critical exponent having most probable value about 1.7 in the three dimensions (must have the universality for various 
percolation problems in the space of same dimensions). $\sigma_{0}$ is some constant.

The value of the critical exponent $t$ for DSG is essentially differ [16]-[20] from the universal value $t_{0}=2$ in percolation theory. This distinction has been explained as the result of tunneling and percolation [16].

Conductivity of DSG has been simulated by the Monte-Carlo method [18] and above mentioned distinction in $t$ values explained as the effect of mishmash of the dopant and the glass particles size.

The tunneling-percolation mechanism [6] [21] [22] Tunneling of charge carriers through the potential barriers compliant to thin glass layer between particles of dopant is the main factor of the conductivity in this model. Since these barriers are connected series with the dopant particles having metallic conductivity, one can write

$$
\rho(T) \approx 0.5 \rho_{\mathrm{b}}(\sin (a T) / a t)(1+\exp (E / k T))+\rho_{\mathrm{d} 0}(1+\alpha T),
$$

here first factor in brackets is the temperature dependence of the tunneling through the barrier, the second one contains very small (about $0.4-5 \mathrm{meV}$ ) energy $E$, which is required to add into or remove the single charge carrier from the dopant particle. $\rho_{\mathrm{b}}$ is the barrier height, $\rho_{\mathrm{d} 0}$ is the resistivity of the dopant particle at $T=0 \mathrm{~K}$, and $k$ is the Boltzmann's constant. Parameter $a$ is the characteristic of the barrier height related to the Fermi level $E_{\mathrm{F}}$, and researchers [6] assume that $a T / 3<1$. The second term in (2) is the resistivity of the dopant particle, $\alpha$ is the temperature coefficient of resistivity of the dopant. It is considered that these barriers and dopant particles form the infinite claster, which connects the terminations of the sample.

Expression (2) has a minimum, caused by metallic conductivity of the dopant particles (second term), but is applicable in the narrow range of temperature. On the other hand, the main distance between the dopant particles in the DSG

$$
L=D \sqrt[3]{\frac{\pi}{6 C}}=D \sqrt[3]{\frac{\pi}{6}\left[1+\frac{\gamma_{\mathrm{d}}}{\gamma_{\mathrm{g}}}\left(\frac{1}{C_{\mathrm{m}}}-1\right)\right]}
$$

is about $0.1-1.5 \mu \mathrm{m}$. Appropriate thickness of the glass layer between the particles is $L-D \approx 0.6 \mu \mathrm{m}$. It is clear that the tunneling of charge carriers is not effective on the same distances, so the description of DSG properties via tunneling runs against the problems. Here $D$ is the diameter of the dopant particles, $\gamma_{\mathrm{d}}$ and $\gamma_{\mathrm{g}}$ are the specific weight of the dopant and the glass accordingly, $C_{\mathrm{m}}$ is the weight content of the dopant. $L \approx 0.87 \mu \mathrm{m}$ for the powder of $\mathrm{RuO}_{2}$ with $D \approx 0.5 \mu \mathrm{m}$ and $C_{\mathrm{m}}=16 \mathrm{wt} \%(C \approx 9.9 \%)$. The resonance tunneling has been used [23] to have a sufficient tunneling probability through the barriers as wide as $0.1 \mu \mathrm{m}$ or larger. This effect assumes the uniform barriers which is hardly probable in the glass having strong structural and chemical inhomogeneities in atomic as well as micrometric scale.

It should be noted also that sinaT/aT in (2) has the first minimum at $a T \approx 4.4$ and the condition $a T / 3<1$ is violated. This minimum slightly shifts towards to lower values of $a T$ due to the second term in (2) while experimental variation of $T_{\mathrm{m}}$ is 77 to $700 \mathrm{~K}$ in accordance with the glass composition, the doping level $C$ and firing temperature $T_{\mathrm{f}}$.

Fluctuation-induced tunneling conduction gives the temperature dependence [24]

$$
\sigma(T)=\sigma_{0} \exp \left[-T_{1} /\left(T+T_{2}\right)\right]
$$

here $\sigma_{0}, T_{1}$ and $T_{2}$ are some parameters. There is no maximum in (4) for $T_{2}>0$ without including the metallic conductivity of dopant relicts and the percolation threshold should be introduced into the model via percolation theory.

Hopping-percolation model [22] [25] is based on the Mott's theory [26] (variable range hopping) and leads to the temperature dependence of conductivity

$$
\sigma(T)=A T^{n} \exp \left[-\left(T_{\mathrm{h}} / T\right)^{1 / 4}\right]
$$

with $T_{\mathrm{h}}=16 a^{3} /\left(k N_{\mathrm{wF}}\right), a$ is the inverse decay distance of electron's spherical wave function (inverse localization distance), $N_{\mathrm{wF}}$ is the density of states of electrons near the Fermi level and $n$ is the constant having most probable value about $1, A$ is some constant value. There is no maximum in (5) as well and hopping of carriers has not the threshold. Because of it hopping of carriers is combined with the percolation theory [27]-[29] as well as the metallic conduction of the dopant particles.

Low-temperature ( $T=0.05-4.2 \mathrm{~K}$ ) measurements show the hopping conduction of the DSG and the decreas- 
ing of the hopping energy due to generation of the narrow impurity band in the forbidden gap of the glass by diffusion of the ruthenium atoms. Concentration of the diffused atoms increases as $T_{\mathrm{f}}$ increases.

There are two problems. The first one caused by the possibility of experimental corroboration of the Mott's low (5). It has been showed [30] that the expression (5) in the generalized form

$$
\sigma(T)=A T^{n} \exp \left[-\left(T_{\mathrm{h}} / T\right)^{m}\right]
$$

can give good agreement with the experimental data in the wide range of $m=0.2-0.55$ if choose the value of $n$ in the interval -7.5 to 2.75 . Despite it the expression (5) with various values of $n$ is used often as the proof of the hopping conductivity in the DSG.

The second problem is the result of the fact that carriers hopping (which is actually the quantum-mechanical tunneling) is not effective in distance about $0.1-1.5 \mu \mathrm{m}$ that corresponded to mean distance between the dopant particles in the DSG. It forces the researchers to consider the diffusion of dopant atoms into the glass or the particles to be far smaller than they are actually.

Authors [31] as well as Shoepe [4] have derive experimentally at low temperatures (0.05 - $2 \mathrm{~K})$ the expression

$$
\sigma(T) \sim \exp \left[-(\Theta / T)^{x}\right]
$$

with $x=0.5$ for DSG conductivity and explained it as result of tunneling though graded barriers. For $x=0.5$ one finds $p=2$ in three dimensions ( $d=3$ ), and this value corresponds to the density of states variations expected to result from Coulomb interaction between localized carriers [28]. Here $d$ is the dimensionality and $p$ is the power by which the density of states $g(E)$ rises about the Fermi level: $g(E)=\left|E-E_{\mathrm{F}}\right|^{p}$. Subsequently the authors [5] have calculated for mentioned experimental results the localization radius $a_{0}=e^{2} /\left(\varepsilon \varepsilon_{0} k T_{\mathrm{h}}\right)$, here $e$ is the electron charge, $\varepsilon_{0}$ is the permittivity of free space, $\varepsilon=10$ is the static dielectric constant of DSG. It was found that $a_{0} \approx 2$ $\mu \mathrm{m}$ and the estimated activation energy less than $k T$. Such value of $a_{0}$ is meaningless because of the mean size of the dopant particles where carriers to be localized is about $0.1-1 \mu \mathrm{m}$. So authors [5] conclude that the percolation theory is not applicable to the DSG.

It was concluded in [32] that hopping mechanism is important at low temperatures and tunneling takes place at high $T$.

Conduction through the narrow impurity band generated in the forbidden band of the glass by diffused dopant atoms due to firing have been considered in [33] [34]. Robertson [34] has proved this model by effect of firing duration $\tau$ and size $D$ of dopant particles on the DSG conductivity. Increasing of $\tau$ results higher concentration of the dopant atoms diffused into the glass and reduces the hopping distance so conductivity increases. But there is no detailed development of this model.

Mismatch of the thermal expansion coefficients of the DSG and the substrate [35] leads to

$$
\rho(T)=\rho_{\Theta}\left\{\exp \left(\frac{\beta}{T}-\frac{\beta}{\Theta}\right)+\psi(T-\Theta) k_{\Theta}[1+\chi(T-\Theta)]\right\},
$$

which takes into account the tension $\psi$ of the DSG layer through the mismatch of thermal expansion coefficients of the glass and the ceramic substrate. Here $\rho_{\Theta}$ is the value of $\rho(T)$ at temperature $\Theta, k_{\Theta}$ is the gauge factor of DSG at $\Theta, \chi$ is the temperature coefficient of the gauge factor. $k \beta$ is the activation energy of DSG.

This mismatch was taken for in [36] as well. It is found that this factor can correct the measured value of temperature coefficient of resistivity on $\pm 100 \times 10^{-6} \mathrm{~K}^{-1}$.

Samples of the DSG glass $+\mathrm{RuO}_{2}$ without the ceramic substrate have been examined in [37]. It is considered that the predominant conductivity is metallic at $C>20 \%$ and ionic at $C<3 \%$. At the intermediate region tunneling and hopping of carriers are coexisted.

Combination of the metallic and thermal activated (semiconducting) conductivity [7] [38] gives

$$
\rho(T)=\rho_{\mathrm{g} 0} \exp \left(E_{\mathrm{a}} / 2 k T\right)+\rho_{\mathrm{d} 0}(1+\alpha T),
$$

which is in the good agreement with experiment at $T>T_{\mathrm{m}}$, but out of keeping at $T<T_{\mathrm{m}}$. First term in (9) is the contribution of the glass layers between the dopant particles having metallic conductivity (the second one).

Prudenziati has pointed out [39] that possible conduction mechanisms of the DSG are hopping, tunneling and transport through the narrow impurity band, formed in the glass interlayers by chemical interactions of the glass 
and dopant.

Effects of firing temperature on the parameters of the DSG have been investigated in [40] and it is concluded that any theoretical models of the DSG conductivity is not adequate to the wide variation range of the DSG parameters. Such conclusion is contained in the number of other articles [31].

Unsoundness of the two-phase system model [6] has been noticed in [12].

Electron microprobe analyses and atomic force microscope investigations [7] [41]-[43] of the interface of glass- $\mathrm{RuO}_{2}$ show that there are the zone of higher concentration of $\mathrm{Ru}$ atoms in the glass round the $\mathrm{RuO}_{2}$ particles. These zones are formed by diffusion of Ru atoms into the glass. It is showed as well [44] that diffusion length of $\mathrm{Ru}$ atoms higher than $1 \mu \mathrm{m}$ and content of them is less than 7 atomic\%. The authors conclude that the conductivity of $\mathrm{RuO}_{2}$ based DSG is realized by hopping of carriers among the Ru clasters of 2 to $4 \mathrm{~nm}$ in size.

Unfortunately, relations of the diffusion zones and the minimum of the temperature dependence of DSG resistivity have not investigated in these works.

\section{Applicability of the Known Models to the $\rho(T)$ of the DSG}

Let us to consider the DSG containing $C_{\mathrm{m}}=16 \mathrm{wt} \%$ of $\mathrm{RuO}_{2}$ and $84 \mathrm{wt} \%$ of lead-silicate glass 2 . $C=9.9 \%$ and the mean distance between centers of spherical particles of the dopant $L=0.87 \mu \mathrm{m}$ for its diameter $D=0.5 \mu \mathrm{m}$ here. DSG is the foil of $l \cdot w \cdot t=10 \times 10 \times 0.025 \mathrm{~mm}^{3}$. Here $w, l, t$ are width, length and thickness of the sample accordingly. $t=0.0025 \mathrm{~cm}$ is the standard thickness of the thick film resistors.

Specific weights are $\gamma_{\mathrm{d}}=6.85 \mathrm{~g} / \mathrm{cm}^{3}$ for the dopant $\left(\mathrm{RuO}_{2}\right)$ and $\gamma_{\mathrm{g}}=4 \mathrm{~g} / \mathrm{cm}^{3}$ for the glass. Resistivity is $\rho_{\mathrm{d}}=4$ $\times 10^{-5} \mathrm{Ohm} \cdot \mathrm{cm}\left(\mathrm{RuO}_{2}\right)$ and $\rho_{\mathrm{g}}>10^{16} \mathrm{Ohm} \cdot \mathrm{cm}$ (the glass). Volume of the sample is $v=2.5 \times 10^{-3} \mathrm{~cm}^{3}$, volume of the dopant is $v_{\mathrm{d}}=C v=0.099 \times 2.5 \times 10^{-3} \approx 2.5 \times 10^{-4} \mathrm{~cm}^{3}$.

We will estimate now the upper and lower limits of the resistivity for the DSG sample. There are two extreme distributions of the dopant particles in the sample (Figure 1). Resistivity of the sample will be intermediate for all other cases.

In the first case the dopant forms the solid film of length $l$ and thickness $t$ along the electric field, i.e. the film closes the metallic terminations (Figure 1(a)). Wideness of the same film is $w_{\mathrm{d}}=C w=0.099 \mathrm{~cm}$ and its resistance is $R_{\mathrm{d} 1}=\rho_{\mathrm{d}} l /\left(w_{\mathrm{d}} \cdot t\right)=4 \times 10^{-5} /\left(0.099 \times 2.5 \times 10^{-3}\right)=0.162 \mathrm{Ohm}$. The resistance of the glass film connected in parallel is $R_{\mathrm{g} 1}=\rho_{\mathrm{g}} \mathrm{l} /\left(\left(1-w_{\mathrm{d}}\right) \cdot t\right)=10^{16} /\left(2.5 \times 10^{-3} \times 0.901\right)=4.4 \times 10^{18} \mathrm{Ohm}$. One can see that the full resistance of the sample $R_{1}=\left(R_{\mathrm{d} 1}^{-1}+R_{\mathrm{g} 1}^{-1}\right)^{-1}=0.162 \mathrm{Ohm}$ is determined by resistance of the dopant film.

In the second case the dopant film is across the electric field (Figure 1(b)) and its resistance is $R_{\mathrm{d} 2}=\rho_{\mathrm{d}} w_{\mathrm{d}} /(w \cdot t)=4 \times 10^{-5} \times 0.099 / 2.5 \times 10^{-3}=1.58 \times 10^{-3} \mathrm{Ohm}$. The resistance of the glass layer in series is $R_{\mathrm{g} 2}=\rho_{\mathrm{g}}\left(1-w_{\mathrm{d}}\right) /(w \cdot t)=10^{16} \times 0.901 / 2.5 \times 10^{-3}=3.6 \times 10^{18} \mathrm{Ohm}$ and the total resistance of the sample $R_{2}=R_{\mathrm{d} 2}+R_{\mathrm{g} 2}=1.58 \times 10^{-3}+3.6 \times 10^{18}=3.6 \times 10^{18} \mathrm{Ohm}$ is determined by the glass film resistance. Accordingly, the temperature dependence of the sample resistance is determined by the dopant layer in the first case and by the glass layer in the second case:

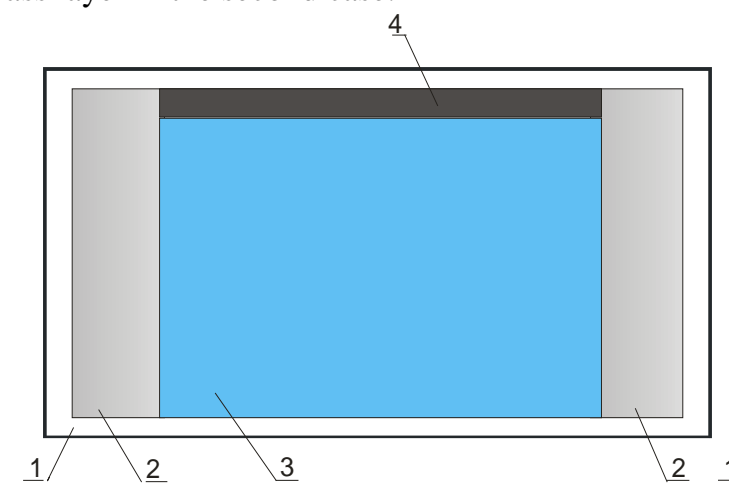

(a)

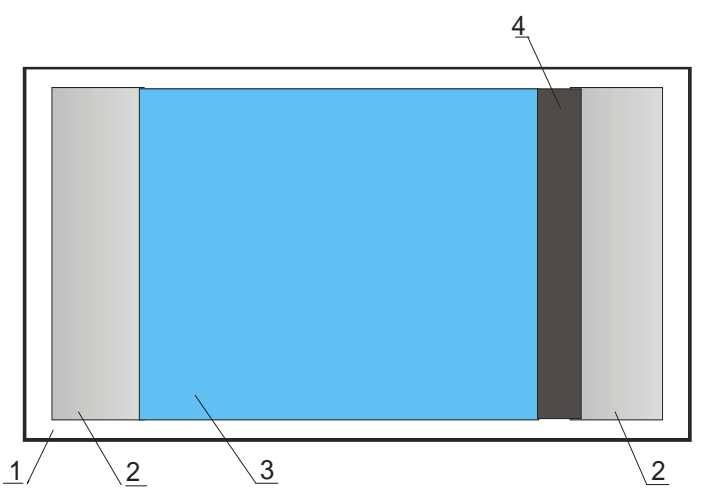

(b)

Figure 1. Configuration of the DSG sample in the cases: dopant layer is along the electric field (a) and across (b). 1 is the substrate, 2 is the metallic terminations, 3 is the glass layer and 4 is the $\mathrm{RuO}_{2}$ layer. 


$$
T C R_{1}=\mathrm{d} R_{1} /\left(R_{0} \mathrm{~d} T\right)=\mathrm{d}(1+\alpha \Delta T) / \mathrm{d} T=\alpha
$$

and

$$
T C R_{2}=\mathrm{d} R_{2} /\left(R_{20} \mathrm{~d} T\right)=-E_{\mathrm{a}} \exp \left(E_{\mathrm{a}} \Delta T / 2 k T_{0} T\right) /\left(2 k T^{2}\right) .
$$

Here $R_{0}$ and $R_{20}$ are values of resistance at some reference temperature $T_{0}, \Delta T=T-T_{0}$ is the deviation of temperature from $T_{0}$. The minimum of the

$$
R(T)=R_{\mathrm{g} 0} \exp (E / 2 k T)+R_{\mathrm{d} 0}(1+\alpha \Delta T)
$$

is possible only if

$$
\alpha>\left|-E_{\mathrm{a}} \exp \left(E_{\mathrm{a}} \Delta T / 2 k T_{0} T\right) /\left(2 k T^{2}\right)\right|
$$

near the minimum.

Simulation of the temperature dependence of the DSG resistance in these cases is shown in Figure 2. In the case of the dopant layer along the electric field, $R(T)$ is linear at low temperatures and has the maximum instead of the experimental minimum so this case is not applicable for DSG.

For this reason we will consider now the metallic and semiconducting sections connected in series (Figure 1(b)) and attempt to find out the correlation between the resistivity values of these sections at the minimum of the total resistivity. For this purpose we rewrite (9):

$$
\rho(T)=a \rho_{\mathrm{g} 0} \exp \left(E_{\mathrm{a}} / 2 k T\right)+(1-a) \rho_{\mathrm{d} 0}(1+\alpha T),
$$

here $a$ and $1-a$ are the contributions of the semiconducting and metallic sections to the total resistivity. The condition of the minimum $\mathrm{d} \rho(T) / \mathrm{d} T=0$ at $T=T_{\mathrm{m}}$ gives $a$ as a function of $T_{\mathrm{m}}$ :

$$
a\left(T_{\mathrm{m}}\right)=\left(1+\frac{1}{\alpha} \frac{\rho_{\mathrm{g} 0}}{\rho_{\mathrm{d} 0}} \frac{E_{\sigma}}{2 k T_{\mathrm{m}}^{2}} \exp \frac{E_{\sigma}}{2 k T_{\mathrm{m}}}\right)^{-1} .
$$

$a\left(T_{\mathrm{m}}\right)$ as function of $T_{\mathrm{m}}$ is showed in Figure 3. It is seen from Figure 3 that $a \approx 0.3-0.9$ is required to have $T_{\mathrm{m}} \approx$ $300 \mathrm{~K}$, as it has often observed experimentally, and $\rho\left(T_{\mathrm{m}}\right)$ must be in order of the $\rho_{g 0}\left(T_{\mathrm{m}}\right) \exp \left(E_{\mathrm{a}} / 2 \mathrm{k} T_{\mathrm{m}}\right)$ as well as $\rho_{d 0}\left(1+\alpha T_{\mathrm{m}}\right)$. But this condition cannot be realized in the DSG, where $\rho_{\mathrm{d}}(300 \mathrm{~K}) \approx 4 \times 10^{-5} \mathrm{Ohm} \cdot \mathrm{cm}$ for $\mathrm{RuO}_{2}$ and $\rho_{\mathrm{g}}(300 \mathrm{~K})>10^{16} \mathrm{Ohm} \cdot \mathrm{cm}$ for the glass $2 \mathrm{SiO}_{2} \cdot \mathrm{PbO}$ while $\rho(300 \mathrm{~K}) \approx 10^{-2}-10^{4} \mathrm{Ohm} \cdot \mathrm{cm}$ for DSG. It means that the resistivity of $\mathrm{RuO}_{2}$ or other dopant particles cannot be used for interpret $\rho\left(T_{\mathrm{m}}\right)$ in terms of the (12) and the physical or chemical mechanism for reducing of the glass resistivity down to $\rho(300 \mathrm{~K})$ should be considered here.

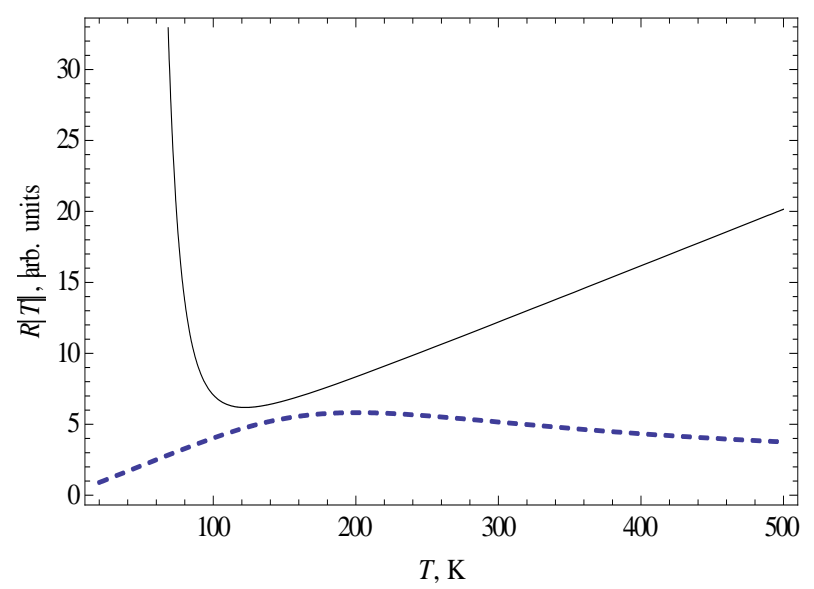

Figure 2. Simulation of the $R(T)$ of the DSG for the dopant layer orientations: along the electric field (dashed line) and across the electric field (solid line). Simulation was carried out for $R(T)=\left((0.1(1+0.4 T))^{-1}+(1.7 \exp (0.45 / k T))^{-1}\right)^{-1}$ and $R(T)=$ $0.1(1+0.4 T)+0.02 \exp (0.45 / k T)$ accordingly. 


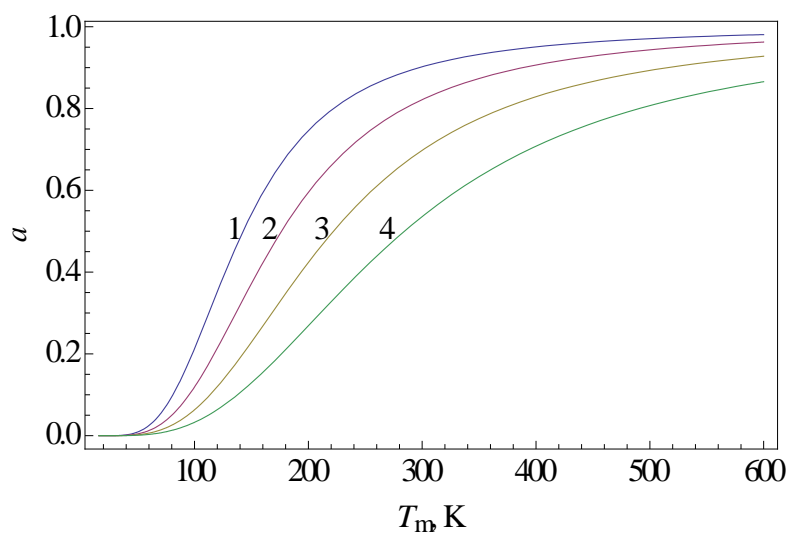

Figure 3. Contribution of the semicon-ducting section $a$ vs. the temperature of minimum $T_{\mathrm{m}}$ for $E_{\sigma}=50 \mathrm{meV}, \alpha=0.004 \mathrm{~K}^{-1}$ and $\rho_{\mathrm{g} 0} / \rho_{\mathrm{d} 0}: 6.7 \times 10^{8}(1) ; 1.35 \times 10^{9}(2) ; 2.7 \times 10^{9}(3) ; 5.4 \times 10^{9}(4)$.

\section{Our Model, Experimental Results and Discussion}

The model and simulations It was pointed out above that the resistivity of the $\mathrm{RuO}_{2}$ used in DSG is lower of the resistivity of the DSG by factor of $10^{-3}-10^{-4}$. As a result the mechanism for lowering the $\rho_{\mathrm{g}}$ from $10^{16}$ $\mathrm{Ohm} \cdot \mathrm{cm}$ down to the $\rho$ of DSG (at least in order of value) should be considered.

Any of the tunneling of free carriers though the glass interlayer or hopping of them from the one dopant particle to another cannot reduce the glass resistivity down to resistivity of the DSG as well as provide the $R(T)$ with the minimum.

Therefore observed properties of the DSG can be caused by doping of the glass interlayer between the dopant particles due to diffusion of the dopant atoms only, and the temperature dependence $\rho(T) \sim T$ or $\rho(T) \sim T^{2}$ should be the property of the doped glass but no that of the $\mathrm{RuO}_{2}$ particles. This condition is satisfied for the electronphonon or the electron-electron scattering.

So let us to consider now the combination of thermal activation, hopping and scattering of charge carriers as the main conduction mechanism of the DSG.

As reported earlier [2] the nanocrystals of silicates in the glass and narrow impurity band formed in firing process can explain the experimental [3] [4] low-temperature resistance of DSG in the form

$$
R(T) \sim T^{-0.5} \exp (K / T)^{\zeta},
$$

if the temperature dependence of the energy gap between the impurity band and the valence band of the glass is taken into account.

It is should be noted that the temperature dependence (13) with $\varsigma=0.5$ takes place in the hopping model of the doped semiconductors as the result of the Coulomb gap in the energy spectrum of the electrons as well [29] but it was noted ibid, that such a consideration is qualified in the crystalline semiconductors and Coulomb gap is not effective in amorphous materials.

We have taken into account therein the temperature dependence of width $E_{\mathrm{G}}(T)$ of the band-gap between the top of the glass valence band and the impurity band bottom caused by electron-phonon coupling [45]-[50]:

$$
E_{\mathrm{G}}(T) \approx E_{\mathrm{G}}(0)-\varsigma T^{2},
$$

and the temperature coefficient $\varsigma$ is about $10^{-6} \mathrm{eV} \cdot \mathrm{K}^{-2}$, so this effect is not essential in the wide-band semiconductors such as $\mathrm{Ge}, \mathrm{Si}, \mathrm{GaAs}$ or $\mathrm{C}$ (diamond). Here $E_{\mathrm{G}}(0)$ is the width of the band gap at $T=0 \mathrm{~K}$.

Expression (14) is the experimental and is not suitable enough at low temperatures $\left(T<\Theta_{\mathrm{D}}\right.$, here $\Theta_{\mathrm{D}}$ is the Debye temperature).

The expression [50]

$$
E_{\mathrm{G}}(T)=E_{\mathrm{G}}(0)-\xi\langle\hbar \omega\rangle(\operatorname{cth}(\langle\hbar \omega\rangle / 2 k T)-1),
$$

is more correctly at $k T<\langle\hbar \omega\rangle$, here $E_{\mathrm{G}}(0)$ is the band-gap width at $T=0 \mathrm{~K}$ (Figure 4), $\xi$ is the electron-pho- 


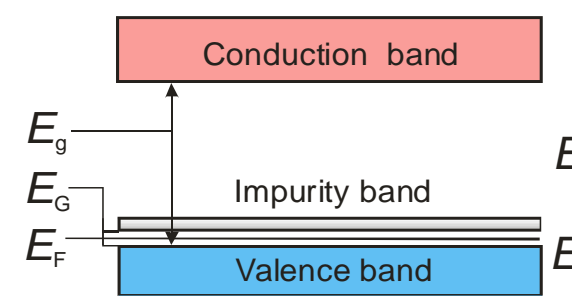

(a)

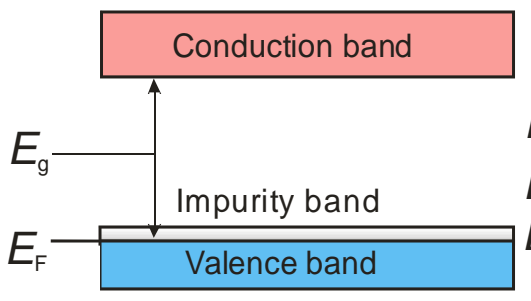

(b)

(c)

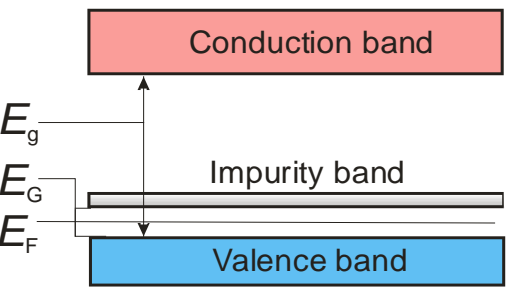

Figure 4. Energy bands and the band-gap $E_{\mathrm{G}}$ in DSG at temperatures $T$ (schematically, K): 0 (a), about 300 (b) and $>900$ (c).

non coupling constant, $\langle\hbar \omega\rangle$ is the main phonon energy in the material. This effect narrows the band gap $E_{\mathrm{G}}$ and it disappears at the certain temperature $T_{\mathrm{m}}$ in the semiconductors having the narrow band gap (less than 10 $50 \mathrm{meV}[51])$ :

$$
\begin{gathered}
E_{\mathrm{G}}\left(T_{\mathrm{m}}\right)=0 . \\
T_{\mathrm{m}}=\frac{\langle\hbar \omega\rangle}{k \ln \left(1+2 \xi\langle\hbar \omega\rangle / E_{\mathrm{G}}(0)\right)} .
\end{gathered}
$$

The impurity band and the valence band merge and form unite partially filled valence band as in the metals (Figure 4) at $T \geq T_{\mathrm{m}}$, so concentration of the charge carriers become constant. The main factor of the temperature dependence of resistivity of the DSG is thereafter the charge carriers scattering on phonons, neutral and ionized impurities or on the other charge carriers, which lead to

$$
\begin{aligned}
& \rho(T) \sim T, \\
& \rho(T) \sim T^{2}
\end{aligned}
$$

or

$$
\rho(T) \sim T^{-3 / 2}
$$

Geometry of the impurity band in the glass band-gap at some characteristic temperatures is shown in Figure 4. Temperature dependence of the impurity band-gap $E_{\mathrm{G}}$ is shown in Figure 5.

Thermal activation and hopping of the charge carriers act simultaneously and increase the DSG conductivity while electron-phonon or electron-electron scattering decreases it so one can write for resistivity of DSG

$$
\rho(T)=\left[\sigma_{\mathrm{a}}(T)+\sigma_{\mathrm{h}}(T)\right]^{-1}+\rho_{\text {met }}(T),
$$

here

$$
\sigma_{\mathrm{a}}(T)=A T \exp \left[-E_{\mathrm{G}}(T) / 2 k T\right]
$$

is the conductivity due to thermal activation of carriers,

$$
\sigma_{\mathrm{h}}=B T^{0.5} \exp \left[-\left(T_{\mathrm{h}} / T\right)^{1 / 4}\right]
$$

is the hopping conductivity, and

$$
\rho_{\text {met }}(T)=\rho_{\mathrm{m} 0}\left(1+\alpha T+\beta T^{2}+\gamma T^{-3 / 2}\right)
$$

is the metallic conductivity, caused by scattering of carriers on neutral defects $\left(\rho_{\mathrm{m} 0}\right)$, phonons $(\alpha T)$, charged impurities $\left(\gamma T^{-3 / 2}\right)$ or other charge carriers $\left(\beta T^{2}\right)$ [52] [53]. The expression (24) assumes that various scattering processes act simultaneously in the DSG.

It should be emphasized that $\rho_{\text {met }}(T)$ is the intrinsic property of the doped glass here in contrary to the (2) and (10), where $\rho_{\mathrm{d} 0}(1+\alpha T)$ is the parameter of the dopant particles. Expression (21) with (15), (22), (23) and (24) is shown in Figure 6.

Experimental results Comparison of the experimental results of the temperature dependence of the resis- 


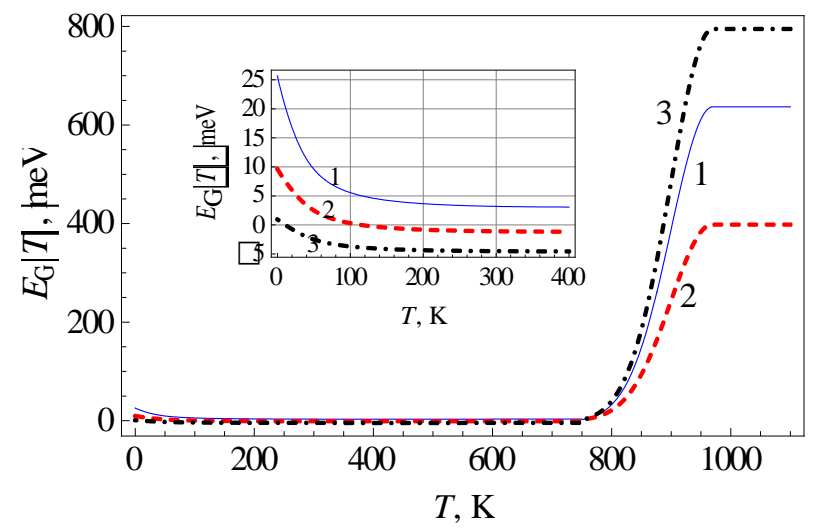

Figure 5. Band-gap width $E_{\mathrm{G}}$ versus temperature including the equation (15) $(T<200 \mathrm{~K})$ and structure transitions of silicate nanocrystals $(T>800 \mathrm{~K})$. Inset is the enlarged part of the main curves. Curves 1, 2 and 3 are responsible for the various width of the band gap at $T=0 \mathrm{~K}$ and beyond the $900 \mathrm{~K}$ (structure transitions have been completed, see [3]).

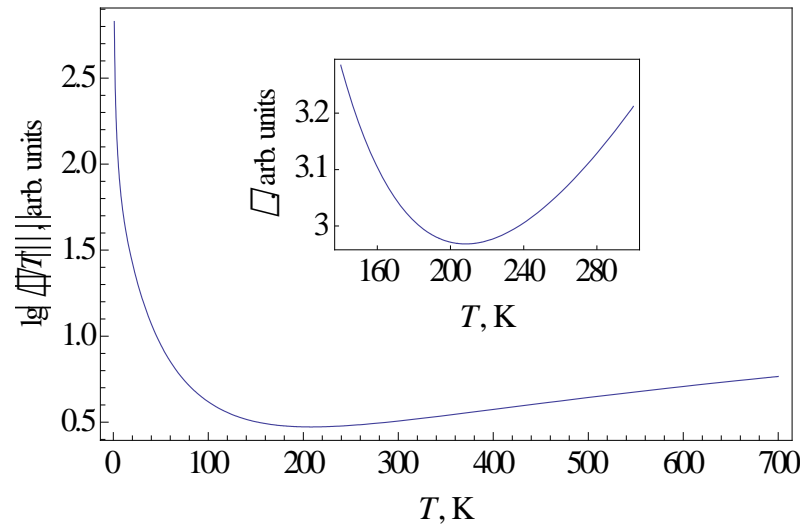

Figure 6. Simulation of the $\rho(T)$ (21) for $A=10^{40} ; B=3.5 ; T_{\mathrm{h}}=$ $100 \mathrm{~K} ; E_{\mathrm{G}}(0)=10^{-2} \mathrm{eV} ;\langle\hbar \omega\rangle=22.5 \mathrm{~K} ; \zeta=0.5$. The inset is the enlarged region near the minimum.

tance $R(T)$ of the DSG samples and our model (21) in the temperature range 160 - $800 \mathrm{~K}$ shows the good agreement (Figures 7-9).

It is seen from these figures that

1) $R(T)$ of the all investigated DSG is not quadratic

$$
R(T)=R_{\mathrm{m}}+B\left(T-T_{\mathrm{m}}\right)^{2},
$$

in contrast with the common opinion [8] [10];

2) The temperature $T_{\mathrm{m}}$ of the minimum is affected by DSG composition and firing conditions $\left(T_{\mathrm{f}}\right.$ and $\left.\tau\right)$;

3) The temperature $T_{\mathrm{m}}$ of the minimum increases with the resistivity $\rho$ of the DSG so can be distorted (Figure 9) by structure transitions of silicate nanocrystals in the DSG [3].

\section{Conclusions}

1) The known conduction mechanisms of the DSG cannot explain the origin of the minimum of the temperature dependence of resistivity and the effect of the glass and dopant composition, firing conditions $\left(T_{\mathrm{f}}\right.$ and $\tau$ ) on its location (temperature $T_{\mathrm{m}}$ and resistivity $\rho_{\mathrm{m}}$ ). 


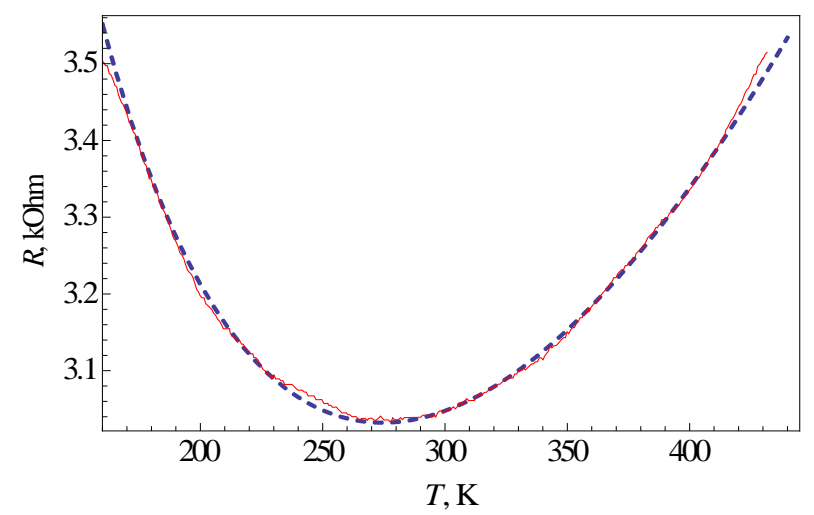

Figure 7. Temperature dependence of resistance of the DSG 1 with $20 \mathrm{w} \% \mathrm{RuO}_{2}$. Dotted line is the function (21) with (15), (21)-(24) for $A=2.5 \times 10^{-7} \mathrm{Ohm}^{-1} \cdot \mathrm{K}^{-1}, E_{\mathrm{G}}(0)=10 \mathrm{meV}, \xi=$ $1.1,\langle\hbar \omega\rangle=2 \mathrm{meV}, B=1.75 \times 10^{-5} \mathrm{Ohm}^{-1} \cdot \mathrm{K}^{-0.5}, T_{\mathrm{h}}=100 \mathrm{~K}, R_{\mathrm{m} 0}=\rho_{\mathrm{m} 0} / t=1.342 \times 10^{3} \mathrm{Ohm}$, $\alpha=0, \beta=3.94 \times 10^{-6} \mathrm{Ohm} \cdot \mathrm{K}^{-2}$.

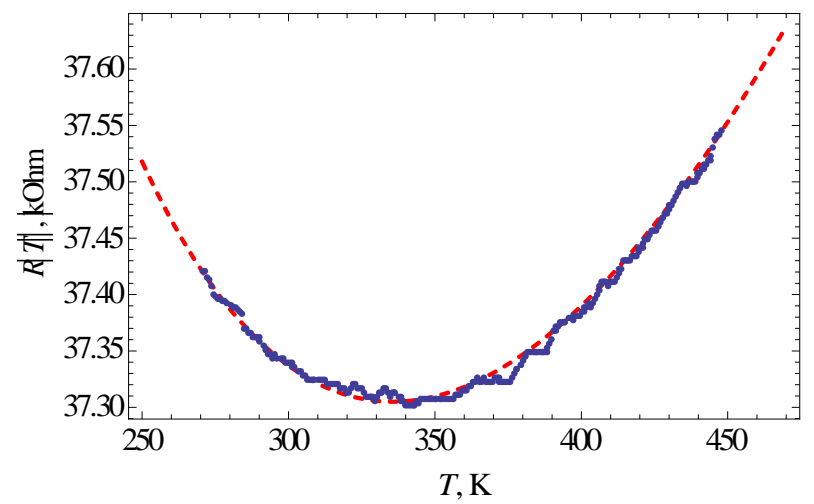

Figure 8. Temperature dependence of resistance of the DSG 3 with $16.5 \mathrm{w} \% \mathrm{RuO}_{2}$. The dotted line is function (21) with (15), (22)-(24) for $A=4.42 \times 10^{-7} \mathrm{Ohm}^{-1} \cdot \mathrm{K}^{-1}, E_{\mathrm{G}}(0)=12.3$ $\mathrm{meV}, \xi=1.1,\langle\hbar \omega\rangle=4.8 \mathrm{meV}, B=3.91 \times 10^{-5} \mathrm{Ohm}^{-1} \cdot \mathrm{K}^{-0.5}, T_{\mathrm{h}}=250 \mathrm{~K}, R_{\mathrm{m} 0}=\rho_{\mathrm{m} 0} / t=$ $34.85 \times 10^{3}$ Ohm, $\alpha=1.2 \times 10^{-5} \mathrm{~K}^{-1}$.

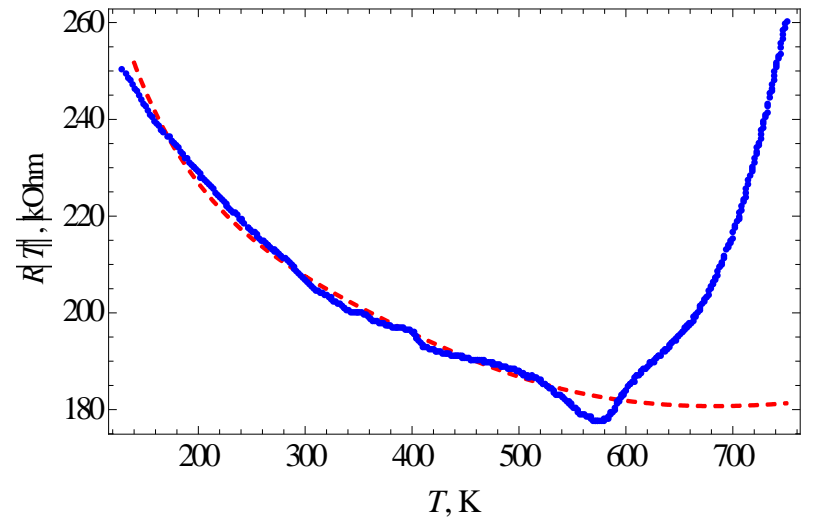

Figure 9. Temperature dependence of resistance of the DSG 2 with $16 \mathrm{w} \% \mathrm{RuO}_{2}$. The dotted line is the function (21) with (15), (22)-(24) for $A=3.27 \times 10^{-8} \mathrm{Ohm}^{-1} \cdot \mathrm{K}^{-1}, E_{\mathrm{G}}(0)=430 \mathrm{meV}$, $\xi=0.01,\langle\hbar \omega\rangle=3.86 \mathrm{meV}, B=4.75 \times 10^{-6} \mathrm{Ohm}^{-1} \cdot \mathrm{K}^{-0.5}, T_{\mathrm{h}}=1050 \mathrm{~K}, R_{\mathrm{m} 0}=\rho_{\mathrm{m} 0} / t=1.58 \times$ $10^{5} \mathrm{Ohm}, \alpha=1.452 \times 10^{-4} \mathrm{~K}^{-1}, \beta=2.952 \times 10^{-5} \mathrm{Ohm} \cdot \mathrm{K}^{-2}$. The minimum is distorted by the resistivity variations due to structure transitions of the silicate nanocrystals in the glass at $T=$ $575 \mathrm{~K}$ and $T>800 \mathrm{~K}$ [3]. 
2) The minimum of the temperature dependence of the DSG resistivity is the result of decreasing of the energy gap between the impurity band and the valence band of the glass by temperature. It is very important here to note that the impurity band-gap is narrow (about tens of meV) as the electron-phonon coupling constant has small value. So the band-gap can vanish at temperature $T_{\mathrm{m}}$ near the room temperature (the broad brand-gap will disappear at high $T_{\mathrm{m}}$ and we can't observe its vanishing due to the structural transitions of nanocrystals [2]). Thereafter the joined partially filled valence band is formed in the DSG, the concentration of the free carriers $n(T)$ becomes constant and the resistivity of the DSG will be affected by temperature due to electron-phonon or electron-electron scattering only so $R(T) \sim T$ or $R(T) \sim T^{2}$ as in the typical metals.

\section{Acknowledgements}

Fund for Support of Fundamental Researches of the Uzbek Academy of Sciences is acknowledged for the financial support (grants 27-10 and 14-12).

\section{References}

[1] Abdurakhmanov, G. (2011) On the Conduction Mechanism of Silicate Glass, Doped by Oxide Compounds of Ruthenium: Diffusion and Percolation Levels. World Journal of Condensed Matter Physics, 1, 19-23. http://dx.doi.org/10.4236/wjcmp.2011.12004

[2] Abdurakhmanov, G. (2011) On the Conduction Mechanism of Silicate Glass Doped by Oxide Compounds of Ruthenium (Thick Film Resistors). 2. Nanocrystals in the Glass and Charge Carrier’s Localization. American Journal Materials Science, 1, 12-17.

[3] Pike, G.E. and Seager, C.H. (1977) Electrical Properties and Conduction Mechanisms of Ru-Based Thick-Film (Cermet) Resistors. Journal of Applied Physics, 48, 5152-5169. http://dx.doi.org/10.1063/1.323595

[4] Schoepe, W. (1990) Conduction Mechanism in Granular $\mathrm{RuO}_{2}$-Based Thick-Film Resistors. Physica, B165 \& 166, 299.

[5] Flachbart, K., Pavlík, V., Tomašovičová, N., Adkins, C.J., Somora, M., Leib, J. and Eska, G. (1998) Conduction Mechanism in $\mathrm{RuO}_{2}$-Based Thick Films. Physica Status Solidi (b), 205, 399-404.

http://dx.doi.org/10.1002/(SICI)1521-3951(199801)205:1<399::AID-PSSB399>3.0.CO;2-X

[6] Smith, D.P.H. and Anderson, J.C. (1980) Electron Conduction in Thick Film Resistors. Thin Solid Films, 71, 79-89. http://dx.doi.org/10.1016/0040-6090(80)90186-8

[7] Abe, A. and Taketa, Y. (1991) Electrical Conduction in Thick Film Resistors. Journal of Applied Physics, 24, 11631171. http://dx.doi.org/10.1088/0022-3727/24/7/022

[8] Prudenziati, M. and Cattaneo, A. (1976) Thermoelectric Power in Thick Film Resistors. Electrocomponent Science Technology, 3, 181-183. http://dx.doi.org/10.1155/APEC.3.181

[9] Inokuma, T. and Taketa, Y. (1987) Control of Electrical Properties of $\mathrm{RuO}_{2}$ Thick Film Resistors. Active and Passive Electronic Components, 12, 155-166. http://dx.doi.org/10.1155/1987/87862

[10] Forlani, F. and Prudenziati, M. (1976) Electrical Conduction by Percolation in Thick-Film Resistors. Electrocomponent Science and Technology, 3, 77-83. http://dx.doi.org/10.1155/APEC.3.77

[11] Bobran, K., Kusy, A., Stadler, A.W. and Wilczynski, G. (1995) Conduction in $\mathrm{RuO}_{2}$-Based Thick Films. International Journal of Electronics, 78, 113-119. http://dx.doi.org/10.1080/00207219508926143

[12] Jiang, J.C., Crosbie, G.M., Tian, W., Cameron, K.K. and Pan, X.Q. (2000) Transmission Electron Microscopy Structure and Platinum-Like Temperature Coefficient of Resistance in a Ruthenate-Based Thick Film Resistor with Copper Oxide. Journal of Applied Physics, 88, 1124-1128. http://dx.doi.org/10.1063/1.373786

[13] Hsieh, Y.H. and Fu, S.L. (1994) A Conduction Model for $\mathrm{BaPbO}_{3}$-Based Thick Film Resistors. IEEE Transactions on Components, Packaging, and Manufacturing Technology, Part A, 17, 316-319. http://dx.doi.org/10.1109/95.296416

[14] Zvyagintsev, O.E., Kolbin, N.I., et al. (1965) Ruthenium Chemistry. In: Zvyagintsev, O.E., Ed., Nauka Publisheing House Moscow. (In Russian)

[15] Lazarev, V.B., Sobolev, V.V. and Shapligin, M.S. (1983) Chemical and Physical Properties of Simple Metal Oxides. Nauka Publisheing House, Moscow. (In Russian)

[16] Vionnet-Menot, S., Grimaldi, C., Maeder, T., Ryser, P. and Strässler, S. (2005) Study of Electrical Properties of Piezoresistive Pastes and Determination of the Electrical Transport. Journal of the European Ceramic Society, 25, 21292132. http://dx.doi.org/10.1016/j.jeurceramsoc.2005.03.018

[17] Vionnet-Menot, S., Grimaldi, C., Maeder, T., Strassler, S. and Ryser, P. (2005) Tunneling-Percolation Origin of Non- 
universality: Theory and Experiments. Physical Review B, 71, Article ID: 064201.

[18] Johner, N. (2009) On the Origin of Transport Non-Universality and Piezoresistivity in Segregated Conductor-Insulator Composites and Application to Thick-Film Resistors. D.Sci. Thesis, École Polytechnique Fédérale DeLausanne, Lausanne.

[19] Grimaldi, C., Maeder, T., Ryser, P. and Strässler, S. (2002) Critical Behavior of the Piezoresistive Response in $\mathrm{RuO}_{2}$-Glass Composites. http://arXiv.org/abs/cond-mat/0212319v1

[20] Grimaldi, C., Ryser, P. and Strässler, S. (2000) Gauge Factor Enhancement Driven by Heterogeneity in Thick-Film Resistors. http://arXiv:cond-mat/0010181v1

[21] de Jeu, W.H., Geuskens, R.W.J. and Pike, G.E. (1981) Conduction Mechanisms and 1/f Noise in Thick-Film Resistors with $\mathrm{Pb}_{3} \mathrm{Rh}_{7} \mathrm{O}_{15}$ and $\mathrm{Pb}_{2} \mathrm{Ru}_{2} \mathrm{O}_{7}$. Journal of Applied Physics, 52, 4128-4134. http://dx.doi.org/10.1063/1.329222

[22] Morten, B., Masoero, A., Prudenziati, M. and Manfredini, T. (1994) Evolution of Ruthenate-Based Thick-Film Cermet Resistors. Journal of Physics D: Applied Physics, 27, 2227-2235.

[23] Aers, G.C. and Lui, H.C. (1990) Theory of Resonant Tunneling through an Array of Quantum Dots. Solid State Communications, 73, 19-21. http://dx.doi.org/10.1016/0038-1098(90)90006-W

[24] Sheng, P. (1980) Fluctuation-Induced Tunneling Conduction in Disordered Materials. Physical Review B, 21, 21802195. http://dx.doi.org/10.1103/PhysRevB.21.2180

[25] Ambegaokar, V., Halperin, B.L. and Langer, J.S. (1971) Hopping Conductivity in Disordered Systems. Physical Review B, 4, 2612-2620. http://dx.doi.org/10.1103/PhysRevB.4.2612

[26] Mott, N.F. and Davis, E.A. (1979) Electron Processes in Non-Crystalline Materials. Clarendon Press, Oxford.

[27] Ziman, J.M. (1979) Models of Disorder. Cambridge University Press, Cambridge.

[28] Shklovskii, B.I. and Efros, A.L. (1984) Electronic Properties of Doped Semiconductors. Springer, Heidelberg. http://dx.doi.org/10.1007/978-3-662-02403-4

[29] Bonch-Bruevich, V.L., Zvyagin, I.P., et al. (1981) Electron Theory of the Disordered Semiconductors. In: BonchBruevich, V.L., Ed., Nauka Publisheing House, Moscow. (In Russian)

[30] Lewis, A.J. (1976) Use of Hydrogenation in the Transport Properties of Amorphous Germanium. Physical Review B, 14, 658-668. http://dx.doi.org/10.1103/PhysRevB.14.658

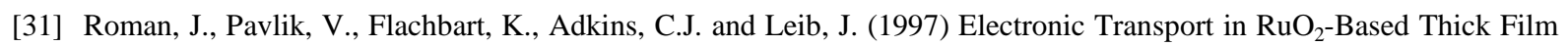
Resistors at Low Temperatures. Journal of Low Temperature Physics, 108, 373-382. http://dx.doi.org/10.1007/BF02397680

[32] Halder, N.C. and Snyder, R.J. (1984) Measurement of the Tunneling and Hopping Parameters in $\mathrm{RuO}_{2}$ Thick Films. ElectroComponent Science and Technology, 11, 123-136. http://dx.doi.org/10.1155/APEC.11.123

[33] Hill, R.M. (1980) Electrical Transport in Thick Film Resistors. ElectroComponent Science and Technology, 6, 141-145. http://dx.doi.org/10.1155/APEC.6.141

[34] Robertson, J. (1977) Conduction Processes in High Value Thick Film Resistors. ElectroComponent Science and Technology, 4, 105-109. http://dx.doi.org/10.1155/APEC.4.105

[35] Sion, R.P., Atkinson, J.K. and Turner, J.D. (1994) A Novel Model for the Temperature Characteristic of a Thick-Film Piezoresistive Sensor. Sensors and Actuators A: Physical, 42, 460-464. http://dx.doi.org/10.1016/0924-4247(94)80034-0

[36] Storbeck, I. and Wolf, M. (1985) On Experimental Data of the TCR of TFRs and Their Relation to Theoretical Models of Conduction Mechanism. ElectroComponent Science and Technology, 11, 255-259. http://dx.doi.org/10.1155/APEC.11.255

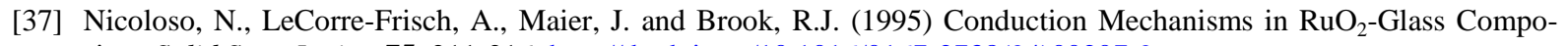
sites. Solid State Ionics, 75, 211-216. http://dx.doi.org/10.1016/0167-2738(94)00207-9

[38] Carcia, P.F., Champ, S.E. and Flippen, R.B. (1976) High Voltage Stable Thick Film Resistors. Proceedings of the 1976 Electronic Components Conference, San-Francisco, 26-28 April 1976, 156-162.

[39] Prudenziati, M. (1983) Electrical Transport in Thick Film (Cermet) Resistors. ElectroComponent Science and Technology, 10, 285-293. http://dx.doi.org/10.1155/APEC.10.285

[40] Lee, J. and Vest, R.W. (1983) Firing Studies with a Model Thick Film Resistor System. IEEE Transactions on Components, Hybrids and Manufacturing Technology, 6, 430-435.

[41] Totokawa, M., Tani, T., Yoshimura, M., Yamashita, S., Morikawa, K., Mitsuoka, Y. and Nonaka, T. (2010) Chemical and Piezoresistive Microanalyses at the Interface of $\mathrm{RuO}_{2}$-Glass Diffusion Pairs. Journal of the American Ceramic Society, 93, 481-487. http://dx.doi.org/10.1111/j.1551-2916.2009.03403.X

[42] Totokawa, M., Yamashita, S., Morikawa, K., Mitsuoka, Y., Tani, T. and Makino, H. (2009) Microanalyses on the 
$\mathrm{RuO}_{2}$ Particle-Glass Matrix Interface in Thick-Film Resistors with Piezoresistive Effects. International Journal of Applied Ceramic Technology, 6, 195-204. http://dx.doi.org/10.1111/j.1744-7402.2008.02325.x

[43] Gabáni, S., Flachbart, K., Pavlík, V., Pietriková, A. and Gabániová, M. (2008) Microstructural Analysis and Transport Properties of $\mathrm{RuO}_{2}$-Based Thick Film Resistors. Acta Physica Polonica A, 113, 625-628.

[44] Adachi, K., Iida, S. and Hayashi, K. (1996) Ruthenium Clusters in Lead-Borosilicate Glass in Thick Film Resistors. Journal of Materials Research, 9, 1866-1878. http://dx.doi.org/10.1557/JMR.1994.1866

[45] Fan, H.Y. (1950) Temperature Dependence of the Energy Gap in Monatomic Semiconductors. Physical Review, 78, 808-809. http://dx.doi.org/10.1103/PhysRev.78.808.2

[46] Fan, H.Y. (1951) Temperature Dependence of the Energy Gap in Semiconductors. Physical Review, 82, 900-905. http://dx.doi.org/10.1103/PhysRev.82.900

[47] Pässler, R. (1996) Comparison of Different Analytical Descriptions of the Temperature Dependence of the Indirect Energy Gap in Silicon. Solid-State Electronics, 39, 1311-1319. http://dx.doi.org/10.1016/0038-1101(96)00037-8

[48] Muto, T. and Oyama, S. (1950) Theory of the Themperature Effect of Electronic Energy Bands in Crystals. Progress of Theoretical Physics, 5, 833-843. http://dx.doi.org/10.1143/ptp/5.5.833

[49] Bansal, K.B., Dixit, V.K., Venkataraman, V. and Bhat, H.L. (2003) Temperature Dependence of the Energy Gap and Free Carrier Absorption in Bulk InAs0.05Sb0.95 Single Crystals. Applied Physics Letters, 82, 4720-4722. http://dx.doi.org/10.1063/1.1587002

[50] O’Donnell, P. and Chen, X. (1991) Temperature Dependence of Semiconductor Band Gaps. Applied Physics Letters, 58, 2924-2926. http://dx.doi.org/10.1063/1.104723

[51] Kerimova, E.M., Gasanov, N.Z., Ismailzade, L.A., et al. (2009) Photoelectric and Optical Properties of the Single Crystals of $\left(\mathrm{TiGaS}_{2}\right)_{1-\mathrm{x}}\left(\mathrm{TlInSe}_{2}\right)_{\mathrm{x}}$ Solid Solutions. Proceedings of the Azerbayjan National Academy of Sciences. PhysicalTechnical Sciences, 5, 137-142.

[52] Abrikosov, A. (1972) Introduction to the Theory of Normal Metals. Academic Press, New York and London.

[53] Kittel, C. (1971) Introduction to Solid State Physics. 4th Edition, John Wiley and Sons Inc., New York, London, Sydney, Toronto. 
Scientific Research Publishing (SCIRP) is one of the largest Open Access journal publishers. It is currently publishing more than 200 open access, online, peer-reviewed journals covering a wide range of academic disciplines. SCIRP serves the worldwide academic communities and contributes to the progress and application of science with its publication.

Other selected journals from SCIRP are listed as below. Submit your manuscript to us via either submit@scirp.org or Online Submission Portal.
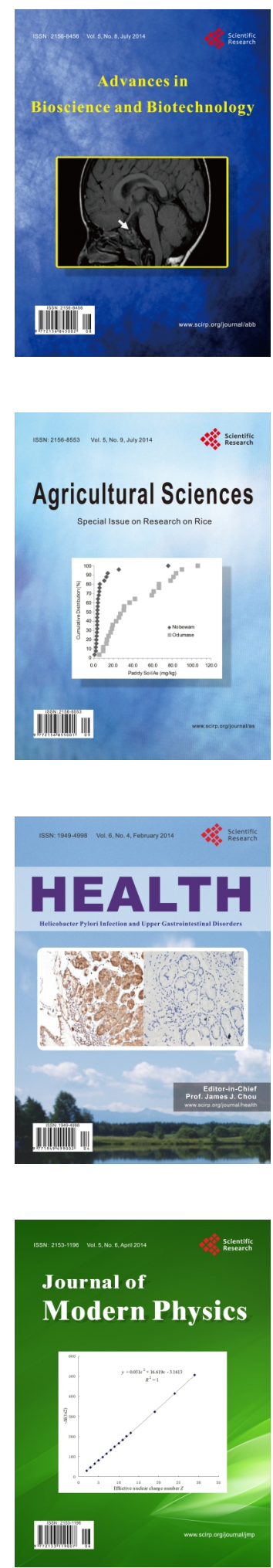
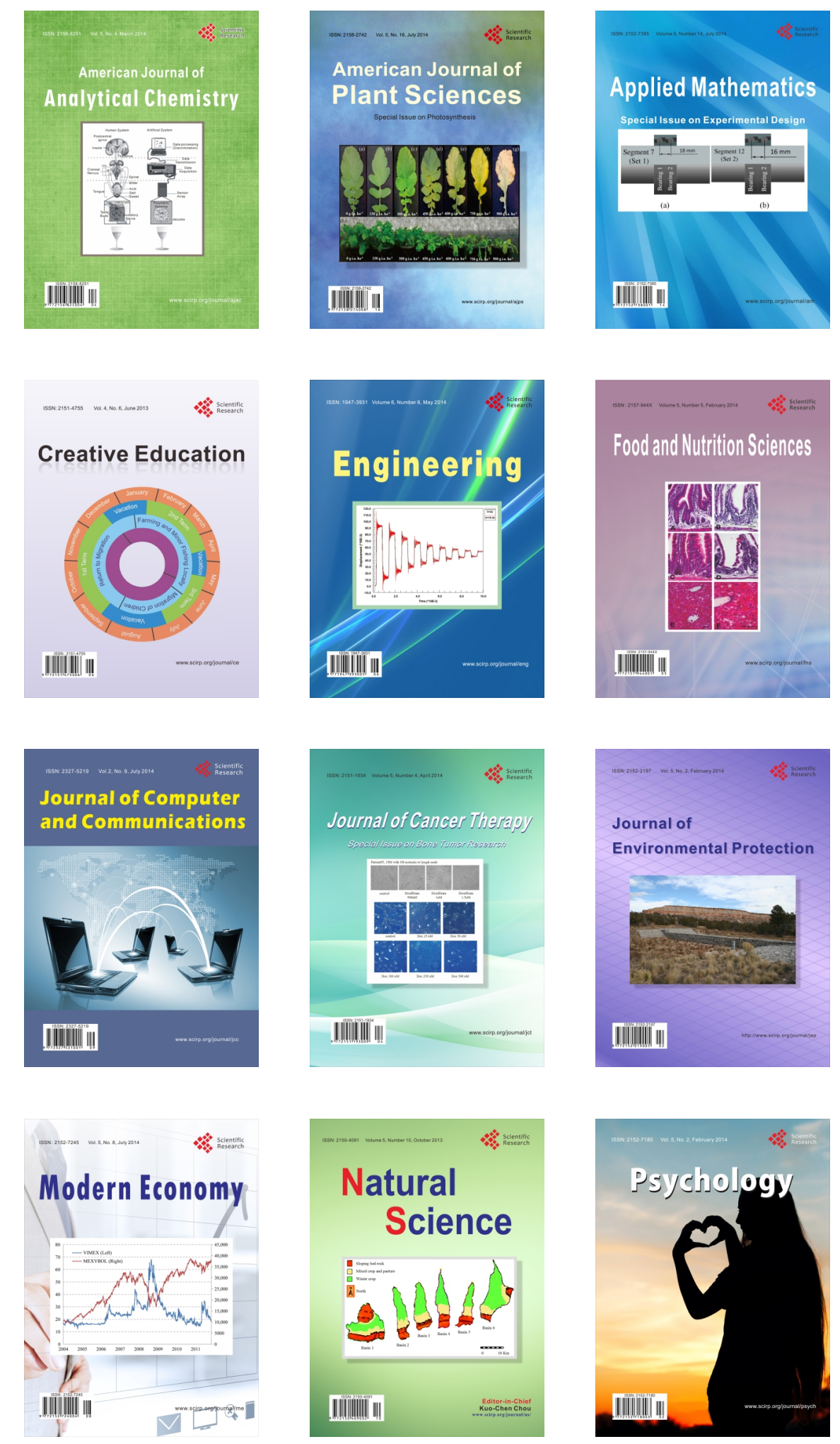